\title{
Towards Transferability of Theories on Prosocial Behavior from Social Psychology to HRI
}

\author{
Barbara Gonsior $^{1,2}$, Malte Buß, Stefan Sosnowski ${ }^{1}$, Dirk Wollherr ${ }^{1,2}$, Kolja Kühnlenz ${ }^{1,2}$, and Martin Buss ${ }^{1,2}$
}

\begin{abstract}
This paper describes the transfer of theories on prosocial behavior from Social Psychology to human-robot interaction (HRI) in terms of helpfulness shown by humans towards a robot. Theoretical foundations are given, and relevant influence factors for prosocial behavior are defined. The paper provides an overview on how these factors can be transferred to HRI and are implemented in two experimental settings. In a first experiment, situational empathy towards a robot is increased. In a second experiment, similarity is induced by means of emotional adaption to the mood of the user. Results show that helpfulness towards a robot can be increased by this approach, thus, re-evaluating the transferability of theories from Social Psychology to HRI.
\end{abstract}

\section{INTRODUCTION}

Most works on social robots are guided by the premise that robots should adapt to humans in order to facilitate intuitive interaction. Nonetheless, proactivity of robots is equally important in order to realize social interaction or to even enable the robot to accomplish its tasks by proactively triggering human behavior. An example are cases where the robot needs the help of humans to achieve a given objective. In the "Interactive Urban Robot (IURO)" project ${ }^{1}$, a social robot is developed, capable of proactively acquiring directional information from humans in order to achieve its objective to navigate to certain goal locations in urban environments, e.g. to perform fetch-and-carry tasks like medicine delivery to its human user. By triggering helpful behavior of humans, IURO is robust against dynamic environmental changes, which can not be pre-programmed. In the context of this project, this work aims to trigger more prosocial human reactions in terms of increased helpfulness towards a robot by transferring social-psychological principles from humanhuman interaction to HRI.

In the following, theoretical foundations on helpful behavior are introduced, and relevant influence factors are defined.

\section{A Robot as Social Actor}

Before transferring theories from Social Psychology to HRI, it is assumed that a robot is perceived by humans as a social actor. In the following, five kinds of cues are described, according to B.J. Fogg [1], that have to be fulfilled to make humans think of robots as social actors:

- Physical cues, like a face, eyes or a body

\footnotetext{
${ }^{1}$ B. Gonsior, M. Buß, S. Sosnowski, D. Wollherr, K. Kühnlenz, and Martin Buss are with Institute of Automatic Control Engineering (LSR), Technische Universität München, D-80290 Munich, Germany bg@tum. de

${ }^{2}$ B. Gonsior, D. Wollherr, and K. Kühnlenz, and Martin Buss are with Institute for Advanced Study (IAS), Technische Universität München, D80290 Munich, Germany

${ }^{1}$ see http://www.iuro-project.eu
}

- Psychological cues, like personal preferences, humor, personality, feelings, empathy, or the ability to apologize

- Language-related cues, including the use of language and recognition of the same

- Social dynamics, like taking turns in a game, cooperation, praising the user for good work, answering questions, and reciprocity, describing the concept of receiving and paying back favors

- Social roles, with the robot being seen as a doctor, teacher or in a similar role

The system used in the experiments is the robotic head EDDIE [2], an emotionally expressive robot head, designed as an interaction partner. The head has 23 degrees of freedom, mixing anthropomorphic (human-shaped) and zoomorphic (animal-shaped) features. By choosing additional animal characteristics, the robot does not provoke disproportionate expectations concerning the social abilities of the robot [3]. Nevertheless, EDDIE shows all of the cues described: It has a face and eyes, and shows feelings through emotional facial and verbal expressions with prosodic variations. It uses language recognition and synthesis, it asks questions during a game, which is used as a communicative person guessing task for the experiments (see Sec. IV). As a social role, it acts as a sort of quiz master during the game. Therefore, it is realistic to assume he will be seen as a humanlike social actor by users.

\section{THEORIES ON HELPFUl BEHAVIOR}

For human-human interaction, helpful behavior and its determinants is a well-studied field of research in Social Psychology [4]. The presented approach is inspired by socialpsychological studies [5], [6], where a feeling of being similar, e.g. in attitudes or characteristics, to a person in need of help turned out to be a motivational activator for increased helpfulness towards this person. This holds true especially in combination with high empathy towards the person, and for situations providing a possibility to avoid helpfulness, e.g. by walking away, referred to as "easy means of escape". More precisely, in case of easy means of escape, the feeling of having something in common with the person in need, paired with correspondingly high empathy, leads to altruistically motivated helpfulness, meaning that the gain in positive stimuli is much higher by helping than by walking away.

In contrast, in the absence of similarity, people would only be highly helpful if there was no or only difficult means of escape. This kind of helpfulness is egoistically motivated in order to reduce one's own discomfort arising from the 
situation. In the case of easy means of escape, people without a feeling of similarity tend to leave the scene, since this is an equally efficient way of reducing the negative stimulus. Empathy would not play a role in this case [7].

It is desirable that increasing helpfulness towards a robot does not restrict the means of escape for users. Therefore, the approach is to raise their motivation to help. As deduced from the social-psychological studies [5], [6], the relevant influence factors for prosocial behavior in terms of increased helpfulness are:

- Empathy, and

- Similarity

Accordingly, the approach is to design the interaction in a way to induce empathy and similarity. To this end, all available output modalities should be used. In this approach, focus is set on emotional facial and verbal expressions.

In the following, it is described how empathy and similarity are applied to HRI in oder to trigger increased helpfulness towards a robot. In a first experiment, situational empathy towards a robot is increased. In a second study, similarity is induced by means of emotional adaption to the mood of the user. Results show that helpfulness towards a robot can be increased by this approach, thus, re-evaluating the transferability of theories from Social Psychology to HRI.

\section{TRANSFER TO HRI}

\section{A. Empathy}

As a means for increasing the amount of empathy, perceived by human users towards a robot, the modality of emotional facial expressions is explored in the context of a communicative person guessing task ${ }^{2}$ [8].

The three tested conditions of facial expressions, shown by the robot, are:

1) Neutral: Display of non-emotional facial expressions

2) Mirror: Display of the same facial expressions, as shown by the human subjects

3) Social Motivation Model (SMM): Display of facial expressions according to an internal model of social variations of smiling [9]-[11], indirectly mirroring the expressions of the human subjects.

After interacting with the robot, the subjects are instructed to rate four statements on situationally induced empathy on a scale from 1 (not true at all) to 5 (completely true).

Results could be deduced from the experimental evaluation including 55 subjects which are distributed equally over experimental conditions. The results showed significantly increased empathy for the SMM condition, compared with animation in a non-adaptive way during the interaction, see Fig. 1.

Since the goal of this approach is to achieve the effect of high helpfulness towards the robot under easy means of escape, as predicted by the above mentioned socialpsychological theories, it is not sufficient to induce high

\footnotetext{
${ }^{2}$ see http://www.akinator.com
}

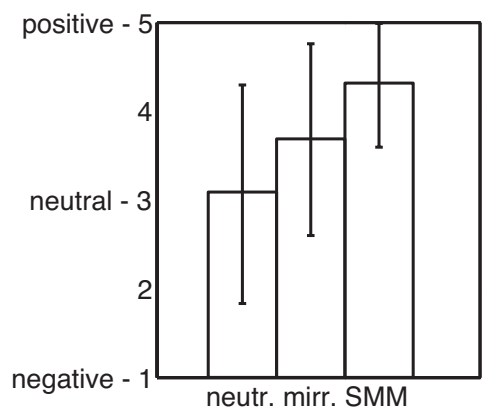

Fig. 1. Situationally induced empathy (on a scale from 1 to 5) and standard deviations for the three experimental conditions [8]

situational empathy towards the robot. Assuming that principles from social psychology are transferable from humanhuman interaction to HRI, a feeling of similarity to the robot, in addition to high situational empathy, is expected to lead to higher helpfulness towards the same, accordingly. Thus, the approach has to incorporate both, the induction of high empathy and similarity.

In the following, it is described how the approach is extended by the induction of similarity between the user and the robot.

\section{B. Similarity}

Since the animation of emotional facial expressions in a socially adaptive way to the user leads to significantly higher results in empathy towards a robot (see SMM in Fig. 1), the approach is enhanced by the experimental induction of similarity: Independent of any interactive goal, the idea is to implement a "social subdialog" in terms of some small talk to open the dialog and thereby monitor the current mood or other personal attitudes of the user. Explicit emotional adaption, and thereby similarity, is created by explicitly stating a mutuality in an attitude or, as applied in the presented study, in the current mood.

\section{Prosocial Behavior}

In order to investigate if the theories on helpful behavior can be transferred from Social Psychology to HRI, both, empathy and similarity, have to be experimentally induced in a user study. Hence, an emotional adaption approach is developed [12] to adapt to the user in two different ways:

- explicit emotional adaption: Prior to the interaction task, the robot adapts to the mood of the user by means of a similarity-statement during a social subdialog.

- implicit emotional adaption: The approach is to use implicit communication channels in a way, adaptive to the user. In this study, the base values of facial and verbal expressions are shifted to the mood of the user, according to an emotional bias, in order to align with the mood of the user as a new starting point for potential emotional variations, e.g. due to task-success or -failure, in the course of the interaction. To this end, the emotional state of the user has to be determined. 
In a first step, this is accomplished by an initial selfassessment by the human participant to be extended by automatic emotion recognition modules in a later stage, such as [22, 36].

As described more detailed in [12], the emotional adaption approach is evaluated, again bound in the interactive person guessing game with the robotic head EDDIE. The tested conditions are:

- Emotional Adaption: In this group, the emotional adaption approach is applied, including explicit and implicit emotional adaption, as described above.

- Comparison Group: In this group, no emotional adaption is applied: During the social subdialog, the robot answers with the utterance "ok" instead of stating that it feels the same way as the user. During the person guessing task, the emotional base values for facial and verbal expressions are not biased by the user mood, but generated according to the task-success or -failure of the robot.

As a measure for helpfulness, the subjects are asked by the robot to help with a picture-labeling task, subsequently to the game. The subjects are free to leave the experiment, and thus are provided with "easy means of escape". The number of pictures labeled, corresponds to the helpfulness towards the robot.

Results can be deduced from the experimental evaluation including 41 subjects, divided randomly onto the two groups. As can be seen in Fig. 2, the participants of the emotional adaption group showed significantly higher helpfulness than the subjects in the comparison group.

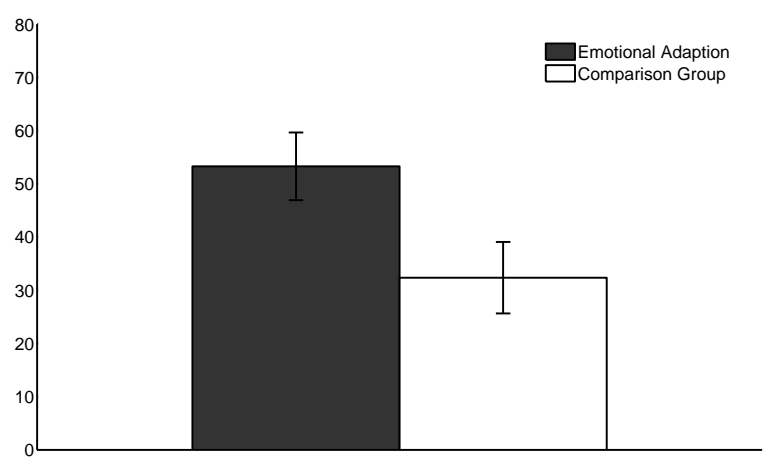

Fig. 2. Helpfulness measure means (on a scale from 0 to 80) and standard deviations according to [12]

\section{Conclusions}

Theories from Social Psychology have been introduced in terms of helpful behavior, and relevant influence factors, namely empathy and similarity, have been designed and transferred to HRI. A methodological approach to trigger more prosocial human reactions in terms of increased helpfulness towards a robot is developed, incorporating both relevant influence factors, and is confirmed by first significant experimental results. Thus, the transferability of theories from Social Psychology can be approved in terms of the targeted helpful behavior.

\section{ACKNOWLEDGMENT}

This work is supported in part by the EU FP7 STREP project "IURO - Interactive Urban Robot)," contract number 248317, see www.iuro-project.eu, the ERC Advanced Grant project "SHRINE - Seamless Human Robot Interaction in Dynamic Environments," contract number 267877, within the DFG excellence initiative research cluster Cognition for Technical Systems - CoTeSys, see www.cotesys.org, and by the Institute for Advanced Study (IAS), Technische Universität München, see also www.tum-ias.de. The authors like to thank Elokence (see www.elokence.com) for providing the interface to the Akinator game (see also akinator.com), and Jürgen Blume for the dialog system.

\section{REFERENCES}

[1] B. Fogg, "Computers as persuasive social actors," Ubiquity, no. 14, 2011.

[2] S. Sosnowski, K. Kuehnlenz, and M. Buss, "Eddie - an emotion display with dynamic intuitive expressions," in Proc. IEEE Int. Symp. on Robot and Human Interactive Communication (RO-MAN), 2006.

[3] K. Kühnlenz, S. Sosnowski, and M. Buss, "Impact of animal-like features on emotion expression of robot head eddie," Advanced Robotics, vol. 24,2010

[4] D. Frey and M. Irle, Theorien der Sozialpsychologie. Verlag Hans Huber, 2002, no. 17

[5] C. D. Batson, B. D. Duncan, P. Ackermann, T. Buckley, and K. Birch, "Is empathic emotion a source of altruistic motivation?" Journal of Personality and Social Psychology, vol. 40, pp. 290-302, 1981.

[6] D. Krebs, "Empathy and altruism," Journal of Personality and Social Psychology, vol. 32, pp. 1134-1146, 1975.

[7] H.-W. Bierhoff, Theorien hilfreichen Verhaltens, 2nd ed. Verlag Hans Huber, 2002, ch. Theorien zu intergruppalem und interpersonalem Verhalten, pp. 178-197.

[8] B. Gonsior, S. Sosnowski, C. Mayer, J. Blume, B. Radig, D. Wollherr, and K. Kühnlenz, "Improving Aspects of Empathy and Subjective Performance for HRI through Mirroring Facial Expressions," in Proc. of IEEE Int. Symp. on Robot and Human Interactive Communication, 2011.

[9] H. Gubler and N. Bischof, "A systems' perspective on infant development. in (eds.),(1-37). hillsdale: Lawrence erlbaum." Infant Development: Perspectives from German-speaking Countries, pp. 1-37, 1990.

[10] I. Borutta, S. Sosnowski, K. Kühnlenz, M. Zehetleitner, and N. Bischof, "Generating artificial smile variations based on a psychological system-theoretic approach," in Proceedings of the 18th IEEE International Symposium on Robot and Human Interactive Communication (Ro-Man), Toyama, Japan, 2009.

[11] B. Gonsior, C. Landsiedel, N. Mirnig, S. Sosnowski, E. Strasser, J. Zlotowski, M. Buss, K. Kühnlenz, M. Tscheligi, A. Weiss, and D. Wollherr, "Impacts of multimodal feedback on efficiency of proactive information retrieval from task-related hri," Journal of Advanced Computational Intelligence and Intelligent Informatics (Special Issue on Cognitive Infocommunications), vol. 16, no. 2, pp. 313-326, 2012.

[12] B. Gonsior, S. Sosnowski, M. Buß, D. Wollherr, and K. Kühnlenz, "An emotional adaption approach to increase helpfulness towards a robot," in Proc. of Int. Conference on Intelligent Robots and Systems (IROS), submitted., 2012. 